\title{
Electron and X-ray Diffraction Measurements of Elastic Stress and Plastic Strain from Ultrasonic Impact Treatment of Aluminum-Magnesium Alloys
}

\author{
L.N. Brewer ${ }^{1,2}$, E.F. Fakhouri ${ }^{2}$, M.E. Haggett ${ }^{2}$, , and K.N. Tran ${ }^{3}$ \\ 1. Metallurgical and Materials Engineering, University of Alabama, Tuscaloosa, AL, USA \\ 2. Mechanical and Aerospace Engineering, Naval Postgraduate School, Monterey, CA, USA \\ 3. Naval Surface Warfare Center Carderock Division, West Bethesda, MD, USA
}

Ultrasonic impact treatment (UIT) is used to prevent fatigue and stress corrosion cracking by placing compressive stresses at the surface of a metallic component. The compressive stresses are generated by severe plastic deformation near the surface and can considerably evolve the microstructure. Mitigation of stress corrosion cracking is particularly important for aluminum-magnesium (Al-Mg) alloys in ship structures as they can sensitize under relatively mild thermal conditions and then experience severe intergranular corrosion and cracking in a marine environment.[1, 2] The connections between UIT processing parameters, plastic strain levels and distributions, and the resultant elastic compressive stresses are not well known, particularly for Al-Mg alloys used in ship structures. In this research, electron backscatter diffraction (EBSD), optical microscopy, and x-ray diffraction were used to determine the connections between impact amplitude and pin diameter on the distribution of plastic and elastic strains in the Al-Mg alloy AA5456.

Four AA5456 plates were gas metal arc (GMA) welded and then ultrasonically treated using a series of | impact displacement levels and pin diameters. Two of the plates were comprised of sensitized material from aging ship structures, while the other two plates were from unsensitized, control material. X-ray diffraction-based, residual stress measurements were performed as a function of distance from the weld for all conditions using cobalt k-alpha x-rays and the Proto Manufacturing iXRD instrument. Crosssectional samples for optical microscopy and EBSD were prepared by cutting the plate perpendicular to the weld and using standard metallographic techniques down to a finish of $0.05 \mathrm{um}$ colloidal silica. For EBSD, the samples were further electropolished using a solution of $90 \mathrm{ml}$ ethanol and $10 \mathrm{ml}$ perchloric acid. EBSD analyses were completed using a Zeiss Neon 40 scanning electron microscope at $20 \mathrm{keV}$ beam voltage, $60 \mu \mathrm{m}$ objective aperture, and a probe current of $1 \mathrm{nA}$. The EBSD signal was collected and analyzed using the EDAX OIM 6.0 software with a Hikari high-speed camera.

The x-ray residual stress profiles show large compressive stresses across the weld, the heat affected zone, and the base metal after UIT (Figure 1). As the pin diameter decreased, the level of compressive residual stress increased, likely because of higher contact pressure for the smaller pins. The displacement amplitude did not affect the level of compressive residual stress. Sensitization level of the alloy also did not have an obvious impact on the level of compressive residual stress.

The distribution of plasticity below the surface varied significantly from one portion of the weld to another (Figure 2). In the fusion zone, the large, annealed grains generated by weld pool solidification were heavily deformed by the UIT process and actually showed a distinct grain refinement at the surface of the UIT crater. This grain refinement after UIT has been observed previously for aluminummagnesium alloys and can actually result in nanocrystalline structures at the very surface of the UIT crater.[3] In the base metal, UIT increased the extent of plastic deformation somewhat over that present from the plate manufacturing, but not to the extent observed in the fusion zone. For the smallest pin diameters and largest displacement level, substantial sub-surface cracking was observed for the 
sensitized plates (Figure 3). This sub-surface cracking was not present in the unsensitized material. From these measurements, it is apparent that all levels of UIT produce substantial compressive stresses, but that an overly large contact stress may result in sub-surface cracking.

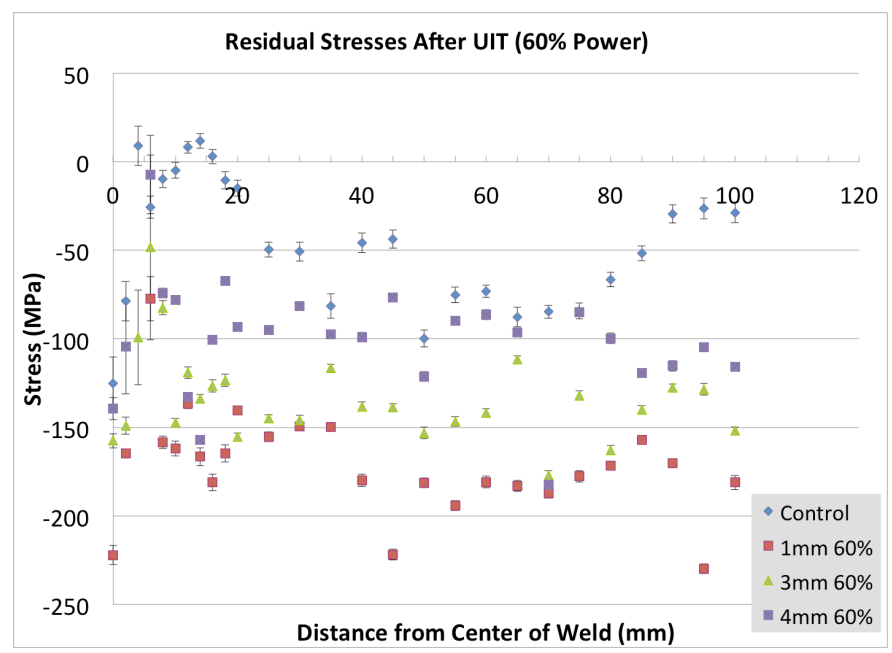

Figure 1. X-ray residual stress profiles for GMA welded aluminum plate after UIT with three different pin diameters. The blue diamonds represent the residual stress profile of the welded sample without UIT.

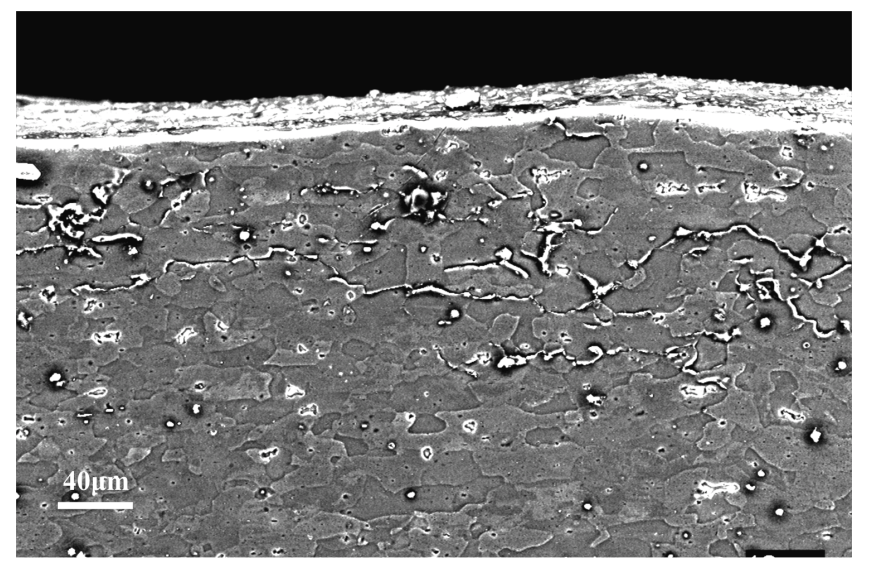

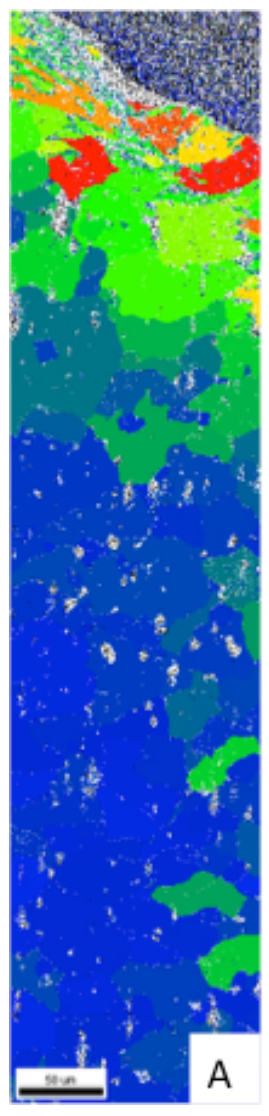

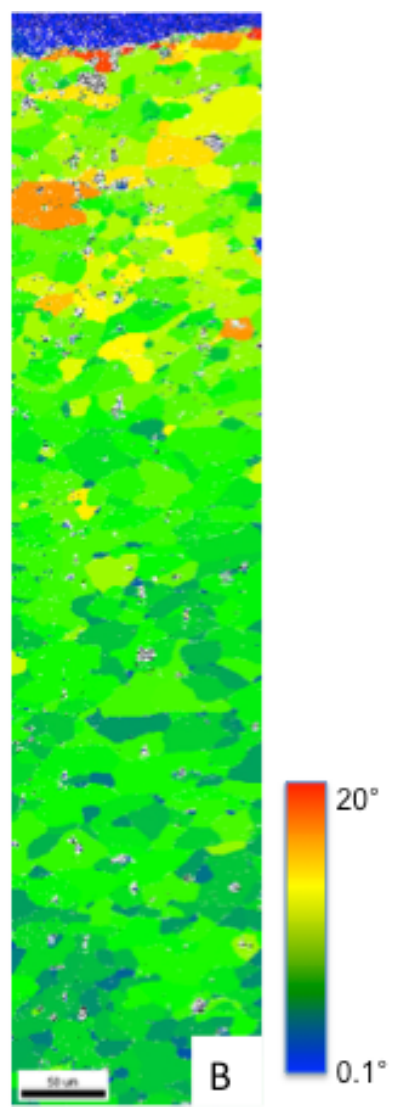

Figure 2. Grain orientation spread maps (GOS) using EBSD for the weld (A) and base metal (B) at the surface (top of map) and subsurface in a pin crater. The color scheme is in degrees. Red denotes an area with a large amount of intragranular misorientation. Blue denotes a region with very little intragranular misorientation.

Figure 3. SEM backscatter image of sub-surface cracking in the heat affected zone of ultrasonically treated, sensitized AA5456 plate ( $1 \mathrm{~mm}$ pin diameter)

\section{References:}

[1] J. Springer et al., Fleet Maintenance and Modernization 2014, American Society of Naval Engineers (2014) p. 1-12.

[2] R. Schwarting et al., Fleet Maintenance and Modernization 2011, American Society of Naval Engineers (2011) p.1-17.

[3] K.N. Tran and L. Salamanca-Riba, Advanced Engineering Materials, 15 (2013) 1105. 